\title{
Pro-apoptotic effects of rHSG on C6 glioma cells
}

\author{
PENG GAO $^{1,2^{*}}$, YOURUI ZOU ${ }^{1 *}$, BING ZHANG $^{1}$, SHUCAI JIANG ${ }^{2}$, WENJIONG HAO $^{4}$, HUI GUO $^{5}$, \\ GUOJIN HUO $^{2}$, JUNCHENG WANG ${ }^{2}$, WEI ZHAO ${ }^{6}$ and BING SHEN $^{1,3}$ \\ ${ }^{1}$ Department of Neurosurgery, General Hospital of Ningxia Medical University; \\ ${ }^{2}$ Graduate School of Ningxia Medical University; ${ }^{3}$ Ningxia Key Laboratory of Cerebrocranial Diseases, \\ Ningxia Medical University, Yinchuan, Ningxia 750004; ${ }^{4}$ Department of Neurosurgery, Yan'an University Affiliated Hospital, \\ Yan'an, Shanxi 716000; ${ }^{5}$ Weifang People's Hospital, Weifang, Shandong 261000; ${ }^{6}$ Medical Science and \\ Technology Research Center, Ningxia Medical University, Yinchuan, Ningxia 750004, P.R. China
}

Received December 21, 2015; Accepted August 17, 2016

DOI: $10.3892 /$ ijmm.2016.2725

\begin{abstract}
Our previous in vitro study demonstrated that the rat hyperplasia suppressor gene (rHSG) inhibited the proliferation of $\mathrm{C} 6$ cells. In the present study, we investigated further the effects of rHSG overexpression on the apoptosis of C6 cells and the possible pathways involved. Hoechst 33342/PI double staining and comet assay were used to examine the morphological characteristics of apoptosis and to examine the effects of rHSG on the apoptosis of the C6 cells. Western blot analysis was used to determine the effects of rHSG overexpression on the protein expression levels of poly(ADP-ribose) polymerase (PARP), cleaved caspase-3, phosphorylated extracellular signal-regulated kinase 1/2 (p-Erk1/2), phosphorylated Akt (p-Akt) and phosphoinositide 3-kinase (PI3K)/Akt, as well as on the mitogen-activated protein kinase (MAPK) pathways induced by insulin-like growth factor (IGF)-1. Our results revealed that the $\mathrm{C} 6$ cells transfected with the rHSG adenoviral vector (Adv-rHSG-GFP group) efficiently expressed rHSG protein; Hoechst 33342/PI double staining and comet assay revealed that rHSG increased C6 cell apoptosis and induced DNA damage. Western blot analysis indicated that rHSG overexpression significantly increased the level of full-length PARP at 24 and $72 \mathrm{~h}(\mathrm{P}<0.01)$, but decreased the level at $48 \mathrm{~h}$ following transfection $(\mathrm{P}<0.01)$, while the proteins levels of cleaved PARP and cleaved caspase- 3 increased significantly $(\mathrm{P}<0.01)$. The protein expression of $\mathrm{p}$-Erk1/2 and $\mathrm{p}$-Akt began to decrease at $48 \mathrm{~h}$ post-transfection $(\mathrm{P}<0.01)$. In addition, the protein levels of Akt and Erk1/2 induced by IGF-1 were significantly inhibited. On the whole, the findings of the present study demonstrate that
\end{abstract}

Correspondence to: Dr Bing Shen, Department of Neurosurgery, General Hospital of Ningxia Medical University, 804 Shengli Street, Yinchuan, Ningxia 750004, P.R. China

E-mail: shenbing315@sina.com

*Contributed equally

Key words: glioma, phosphoinositide 3-kinase/Akt, mitogen-activated protein kinase, rat hyperplasia suppressor gene, apoptosis, C6 cells
rHSG overexpression induces the apoptosis of rat glioma cells, and that these effects may involve the PI3K/Akt and MAPK pathways.

\section{Introduction}

Glioma is the most common primary central nervous system tumor, which accounts for approximately $50 \%$ of all neuroepithelial tumors (1). The 5-year survival rate of patients with glioma is generally $<50 \%$. The median survival time is $<1$ year, and the majority of patients with this tumor succumb to the disease within 2 years following diagnosis $(2,3)$, particularly those with glioblastoma multiforme (GBM), which is a highly malignant tumor with a high post-operative recurrence rate (4). According to the statistics of WHO in 1998, malignant glioma is the second leading cause of death for all patients, the second leading cause of death for tumor patients aged $<34$ years, and the third leading cause of death for tumor patients between the ages of 35-54 years (5). Current opinions suggest that the development and progression of glioma is a complex, multistep process with multiple genes involved.

The activation of multiple cell signaling pathways has been demonstrated to be involved in the development and progression of glioma, among which the vascular endothelial growth factor (VEGF) and Ras pathways have been widely considered to be involved (6-8). In recent years, some information has already been obtained regarding the pathogenesis and treatment of malignant glioma (9). However, the mechanisms involved in the invasive growth and infinite proliferation remain unclear. Further investigations of the pathogenesis of malignant glioma and search for novel treatment methods are of great importance for reducing the mortality associated with malignant glioma.

Over the past decade, the efforts in the development of more effective, novel gene therapies for the treatment of GBM have resulted in some pre-clinical information and in many promising gene therapies. The rat hyperplasia suppressor gene (rHSG), also known as the rat mitofusin-2 (rMfn2) gene, is a newly discovered gene. rHSG was found firstly in vascular smooth muscle cells (VSMCs) in hypertensive rats. The expression of rHSG has been shown to be significantly downregulated in the VSMCs of hypertensive rats compared 
with normal rats (10). It has been demonstrated that $\mathrm{rHSG}$ regulates the apoptosis of VSMCs via the mitochondrial apoptotic pathway (11). In vitro and in vivo studies have also demonstrated that the abnormal cell apoptosis and invasion during tumorigenesis is closely associated with the activation of extracellular signal-regulated kinase (Erk) (12-15). Therefore, inhibiting the activity of Erk may effectively inhibit tumor invasion and may promote cell apoptosis (16-19). A previous study demonstrated the evident disruption of the constitutively activated phosphoinositide 3-kinase (PI3K)/Akt signaling pathway in malignant glioma (20). However, to the best of our knowledge, no study on the effects of $\mathrm{rHSG}$ on the $\mathrm{PKCa} / \mathrm{mitogen}$-activated protein kinase (MAPK) and PI3K/Akt pathways in glioma has been published to date.

Our previous in vitro study demonstrated that the overexpression of rHSG suppressed the proliferation of rat glioma cells, based on the findings that the protein expression level of rHSG was higher in the C6 cells in the group infected with Adv-rHSG-GFP; cell cycle was arrested at the G0/G1 phase, and rat glioma cell proliferation was markedly inhibited; the expression of $\mathrm{p} 27^{\mathrm{Kip} 1}$ and $\mathrm{p} 21^{\mathrm{Cip} 1}$ was increased, while the expression of PCNA was decreased (21).

In the present study, we investigated the effects of rHSG on the apoptosis of $\mathrm{C} 6$ rat glioma cells and the roles of the protein kinase $\mathrm{C}(\mathrm{PKC}) \alpha / \mathrm{MAPK}$ and PI3K/Akt pathways.

\section{Materials and methods}

Materials. C6 cells (Type Culture Collection of the Chinese Academy of Sciences, Shanghai, China) and purified Adv-rHSG-GFP virus-containing solution (titer, $1 \times 10^{11} \mathrm{pfu} /$ $\mathrm{ml}$ ) were preserved in our laboratory. Purified Adv-GFF virus-containing solution (titer, $1 \times 10^{11} \mathrm{pfu} / \mathrm{ml}$ ) was purchased from Baosai Biological Technology Co., Ltd. (Beijing, China). Fetal bovine serum (FBS), Dulbecco's modified Eagle's medium (DMEM; high glucose), trypsin (0.25\%), penicillin and streptomycin were purchased from HyClone (Logan, UT, USA). Insulin-like growth factor (IGF)-1 was purchased from Sigma-Aldrich (St. Louis, MO, USA). Mouse-anti-rat rHSG monoclonal antibody (Cat. no. ab56889) was purchased from Abcam (Cambridge, MA, USA); rabbit-anti-rat monoclonal antibodies to poly(ADP-ribose) polymerase (PARP; Cat. no. 9542S), caspase-3 (Cat. no. 9664S), phosphorylated (p-)Akt (Cat. no. 9271S), Akt (Cat. no. 9272S), p-Erk1/2 (Cat. no. 9101S) and Erk1/2 (Cat. no. 9102S) were purchased from Cell Signaling Technology, Inc. (Danvers, MA, USA). Mouse-anti-rat $\beta$-actin polyclonal (Cat. no. TA-09) and goat-anti-mouse IgG (Cat. no. ZB-2305) antibodies, the immunohistochemical staining kits and DAB solution were purchased from Zhongshan Jinqiao Biotechnology Co., Inc. (Beijing, China). The Hoechst 33342/PI double staining kits, comet assay kits, total protein extraction kits, and BCA protein quantification kits were purchased from Kaiji Biotechnology Co., Ltd. (Nanjing, China). ECL luminol solution was purchased from Pierce Biotechnology, Inc. (Rockford, IL, USA).

Cell culture. C6 cells were thawed, and DMEM culture medium containing $10 \% \mathrm{FBS}$ was added, and the cells were then incubated at $37^{\circ} \mathrm{C}, 5 \% \mathrm{CO}_{2}(\mathrm{v} / \mathrm{v})$ in an incubator with constant humidity. Following adhesion, DMEM medium containing $0.2 \%$ FBS was used to synchronize the cells for $24 \mathrm{~h}$. Afterwards, adenovirus with optimal multiplicity of infection (MOI) was used to transfect the cells (PBS was used for the PBS control group) for $4 \mathrm{~h}$, and the culture medium was then discarded; then the cells were continuously cultured with fresh full culture medium until use in the subsequent experiments.

Transfection of C6 cells with adenovirus. The C6 cells were divided into 3 groups, as follows: the PBS control, Adv-rHSG-GFP and Adv-GFP groups. The cells were seeded into a 6-well plate at a density of $1 \times 10^{5}$ cells/well, and then Adv-rHSG-GFP or Adv-GFP at an MOI of 0, 10, 50, 80 and 100 was added to transfect the cells. An inverted fluorescence microscope (Olympus TL-4; Olympus Optical Co., Ltd., Tokyo, Japan) and a flow cytometer (BD FACSCalibur; BD Biosciences, San Jose, CA, USA) were used to measure the MOI at the optimal transfection efficiency $24 \mathrm{~h}$ later. Green-stained cells were considered positive cells that had been successfully transfected. The transfection efficiency was considered high if $90 \%$ of the cells exhibited green fluorescence. The cells were collected at $24,48,72$ and $96 \mathrm{~h}$ post-transfection for use in apoptosis assay.

Measurement of cellular rHSG expression by immunocytochemistry. The C6 cells were seeded into a 24-well plate pre-coated with coverslips at a density of $5 \times 10^{4}$ cells/well and transfection was carried out following culture for $24 \mathrm{~h}$. The cells were collected at different time points $(24,48,72$ and $96 \mathrm{~h}$ ), and immunocytochemistry was then performed using immunohistochemical staining kits according to the manufacturer's instructions. Following visualization with DAB, contrast staining with hematoxylin, and dehydration with gradient ethanol, the slides were dried and mounted. The C6 cells with a brown-yellow or dark brown stained cytoplasm were considered as rHSG-positive. Upright microscope (Olympus BH2-RFCA) was used to obtain images at a magnification of $x 400$. The images were imported to an image analysis system (Image-Pro Plus 6.0) to calculate the mean optical density of the positive areas on each slide (IOD/area), which was used as the index to reflect the positive strength of rHSG.

Determination of IGF-1 concentration and stimulation of C6 cells. The C6 cells were seeded into 60-mm culture dishes at a density of $1 \times 10^{6}$ cells/well, and $10 \%$ DMEM medium containing $0,1,5,8$ and $10 \mathrm{ng} / \mathrm{ml} \mathrm{IGF-1} \mathrm{was} \mathrm{used} \mathrm{to} \mathrm{stimulate}$ the C6 cells for 15 min following culture for $24 \mathrm{~h}$. Western blot analysis was applied for analyzing the expression level of p-Akt and p-Erk1/2 in order to determine the optimal concentration. By using the same method described above, the cells were stimulated with IGF-1 at the optimal concentration for 0 , $5,15,30$ and 60 min to determine the optimal stimulating time. Subsequently, the optimal concentration and treatment time for stimulation with IGF-1 would be applied to the relevant experiment.

Measurement of the protein expression of rHSG, PARP, cleaved caspase-3, $p$-Akt and p-Erkl/2 by western blot analysis. The C6 cells were seeded into 60-mm culture dishes at a density of $1 \times 10^{6}$ cells/well, and treatments were then carried out. The cells were collected at different time points $(24,48$, 
72 and 96 h). Proteins were extracted from the cells using the total protein extraction kits according to the manufacturer's instructions, and the BCA method was used to determine the protein concentration. The appropriate volume of the protein sample was added to loading buffer, after boiling at $100^{\circ} \mathrm{C}$ for 6 min to allow denaturation. SDS-polyacrylamide gel electrophoresis (SDS-PAGE) was then performed, and the proteins were then transferred to a polyvinylidene fluoride (PVDF) membrane (Millipore Co., Millipore, Billerica, MA, USA) and blocked with 5\% skim milk powder. Primary antibody was then added, followed by incubation at $4^{\circ} \mathrm{C}$ overnight. The primary antibodies used in the present study include the antibodies against rHSG (1:400), PARP $(1: 1,000)$, caspase-3 $(1: 1,000)$, p-Akt (1:500), p-Erk1/2 (1:500) and $\beta$-actin $(1: 1,000)$. The membrane was then washed 3 times with TBST, after which the corresponding secondary antibodies were added. Subsequently, the chemiluminescence method was used to evaluate the relative protein level of rHSG.

Measurement of C6 cell apoptosis buy Hoechst 33342/PI double staining. The C6 cells were seeded into a culture dish with a glass bottom that is specifically used for a laser scanning confocal microscope at a density of $1 \times 10^{5}$ cells/well. Following treatment, the cells in each group were washed with PBS once, and then $5 \mu \mathrm{l}$ of Hoechst 33342 was added and mixed gently. The cells were then placed in the dark at room temperature for 10 to $15 \mathrm{~min}$. PBS was then used to wash the cells once. PI solution $(5 \mu \mathrm{l})$ was added after washing the cells with PBS for an additional 2 times, followed by gentle mixing. The cells in the respective groups were incubated in the dark at room temperature for a further 10-15 min. A laser scanning confocal microscope (LSCM; FV1000 IX81; Olympus) was used to observe the cells at a magnification of x400 immediately after another wash with PBS, and bi-channel images were then obtained with Hoechst (excitation wavelength of $350 \mathrm{~nm}$ ) and PI staining (excitation wavelength of $488 \mathrm{~nm}$ ).

Determination of DNA damage in C6 cells induced by rHSG by comet assay. The C6 cells were collected at different time points $(24,48,72$ and $96 \mathrm{~h})$ and were resuspended with PBS to obtain a density of $1 \times 10^{6}$ cells $/ \mathrm{ml}$. The slides were covered with 3 layers of agarose gel, the upper and lower layers with $0.5 \%$ normal melting point (NMP) agarose and the middle layer with $0.7 \%$ low melting point (LMP) agarose with $10^{4} \mathrm{C} 6$ cells. The slides with agarose gel were placed in cell lysis solution at $4^{\circ} \mathrm{C}$ for $24 \mathrm{~h}$. They were then rinsed with PBS and immersed in fresh alkaline electrophoresis buffer at room temperature for $1 \mathrm{~h}$ to allow DNA unwinding. Electrophoresis was carried out for $20 \mathrm{~min}$ at $25 \mathrm{~V}$, and the slides were then placed in a dish; $0.4 \mathrm{~mm} / 1$ Tris- $\mathrm{HCl}$ solution (PH 7.5) was added for $10 \mathrm{~min}$. Each slide was stained with $20 \mu \mathrm{l}$ PI and covered with a coverslip. The slides were placed in the dark for $10 \mathrm{~min}$ to allow staining, and an inverted fluorescence microscope (Olympus TL-4; Olympus) was then used for observation and imaging. The cells with comet tails formed by the migration of DNA from the nuclei were considered to have DNA damage.

Statistical analysis. SPSS 19.0 statistical software was used for the statistical analysis. All the experiments were repeated at least 3 times. Quantitative data are expressed as the means \pm standard deviation (SD) ( $\mathrm{n} \geq 3$ experiments); one-way analysis of variance (ANOVA) was used for comparing the means among different groups. A value of $\mathrm{P}<0.05$ was considered to indicate a statistically significant difference.

\section{Results}

Effecient transfection of C6 cells with adenovirus. An inverted fluorescence microscope was used to observe the C6 cells at $24 \mathrm{~h}$ post-transfection, and it was found that the percentage of positive cells with GFP expression increased with the increasing MOI (Fig. 1C and D). After obtaining the results using the microscope, the cells were collected and measured using a flow cytometer. The results revealed that when the MOI was 100, the transfection efficiency was $>90 \%$ (Fig. 1A and B). The C6 cells that were transfected with Adv-rHSG-GFP at an MOI of 100 were transiently fixed with paraformaldehyde. DAPI was used to stain the nuclei, and a fluorescence microscope was then used to estimate the transfection efficiency (Fig. 1E). Therefore, the following experiments were carried out on the cells transfected with the virus at an MOI of 100 .

High protein expression level of rHSG in C6 cells. Immunocytochemistry revealed that $\mathrm{rHSG}$ protein was expressed in the cytoplasm of the C6 cells, which was brownyellow in color (Fig. 2A). The protein expression of rHSG was significantly higher in the Adv-rHSG-GFP group compared with the control group or the Adv-GFP group $(\mathrm{P}<0.01, \mathrm{n}=6$ experiments; Fig. 2C). The results of western blot analysis indicated the rHSG-specific band at each time point (Fig. 2B); the expression of rHSG was significantly higher in the Adv-rHSG-GFP group compared with the Adv-GFP and control groups $(\mathrm{P}<0.01, \mathrm{n}=3$; Fig. 2D). These results demonstrated that the transfection of $\mathrm{C} 6$ cells with Adv-rHSG-GFP resulted in a high protein expression level of rHSG.

Apoptosis of the C6 cells induced by rHSG. A laser scanning confocal microscope was used to observe the cells, and it was found that the C6 cells in the Adv-rHSG-GFP group exhibited distinct apoptosis-defining morphological changes at 24-96 h post-transfection. The cells exhibited chromosome condensation, karyopyknosis and nuclear fragmentation. In addition, the nuclei of the cells were deeply stained and presented with a brilliant blue color. The chromatin gradually aggregated adjacent to the nuclear membrane in the shape of a crescent moon. However, for the cells in the control and Adv-GFP groups, the nuclei exhibited homogenously distributed weak dark-blue fluorescence, and no sign of apoptosis was observed (Fig. 3).

The C6 cells in the Adv-rHSG-GFP group were collected at 24-96 $\mathrm{h}$ post-transfection, and the results of comet assay indicated that the DNA of the cells had migrated from the nuclei and formed evident comet tails, suggesting the presence of DNA damage. However, no evident comet tails were observed in the control group or the Adv-GFP group (Fig. 4).

The results of western blot analysis also revealed that the protein expression of full-length PARP increased significantly at 24, 72 and $96 \mathrm{~h}$ post-transfection in the Adv-rHSG-GFP group $(\mathrm{P}<0.01, \mathrm{n}=3)$; however, the expression level decreased 

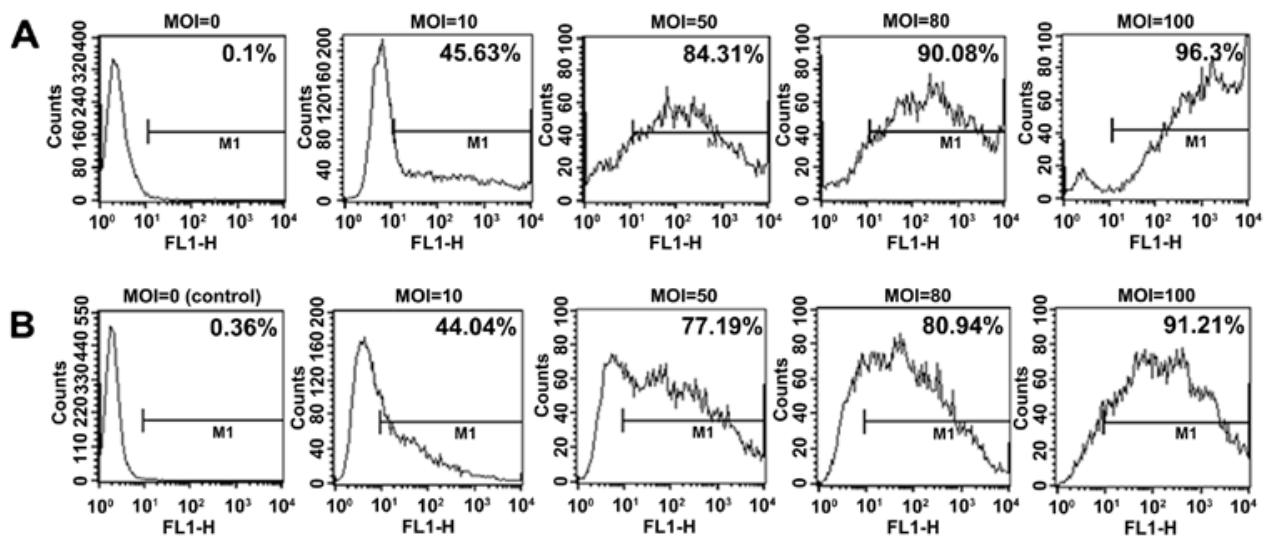

C
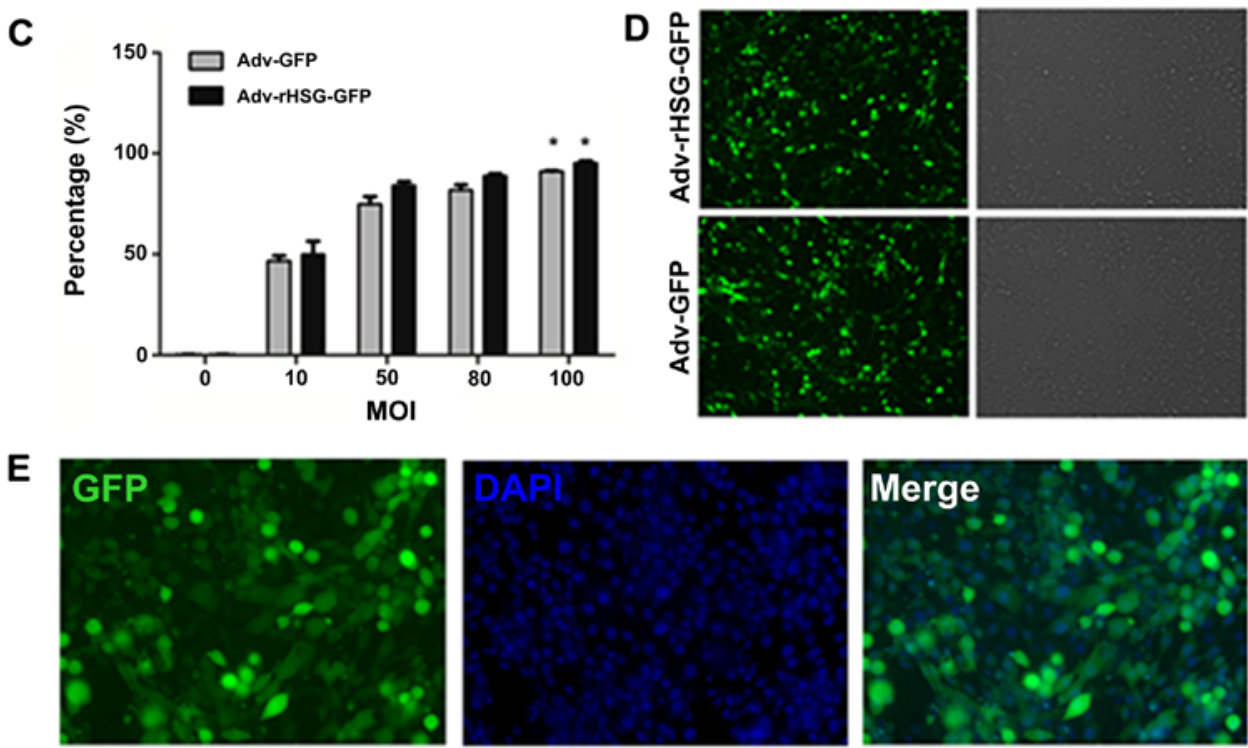

Figure 1. Measuring the efficiency of transfecting C6 cells with adenovirus. (A) Measuring the efficiency of transfecting the cells with Adv-rat hyperplasia suppressor gene (rHSG)-GFP using a flow cytometer; (B) measuring the efficiency of transfecting the cells with Adv-GFP using a flow cytometer; (C) histogram showing the transfection efficiency; (D and E) observing the cells transfected with Adv-rHSG-GFP or Adv-GFP at a multiplicity of infection (MOI) of 100 with a fluorescence microscope ( $\mathrm{x} 400$ magnification). $\mathrm{P}<0.01$, compared with the control group.

A

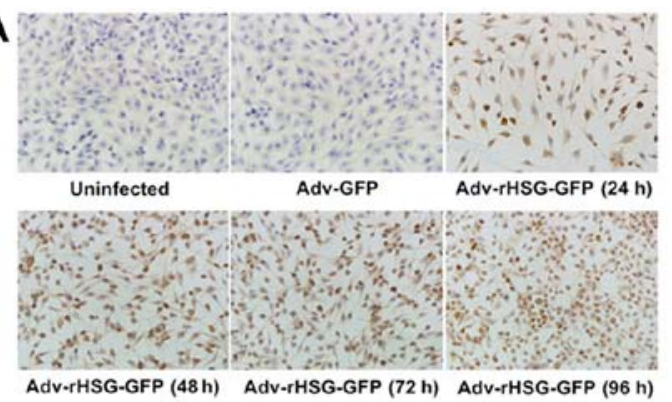

C

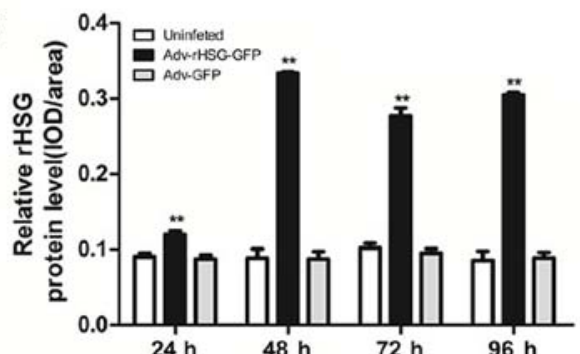

B

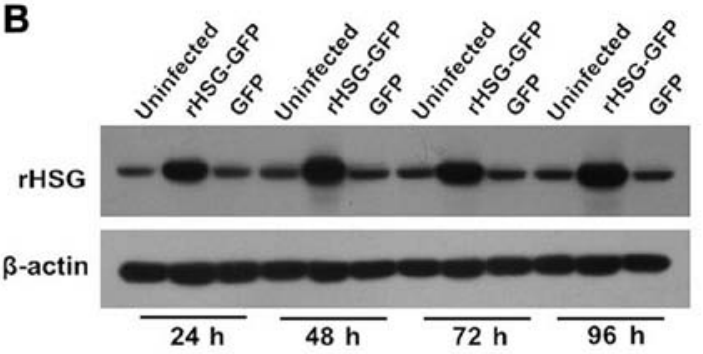

D

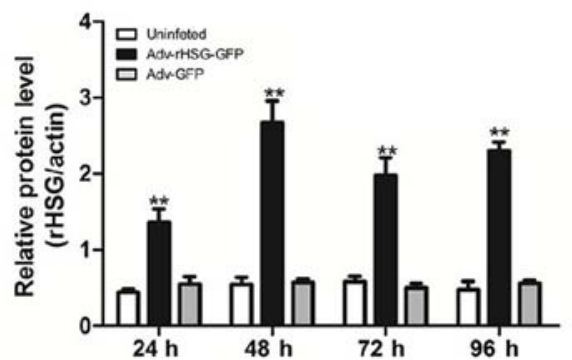

Figure 2. Protein expression of rat hyperplasia suppressor gene (rHSG) at different time points after the C6 cells were transfected with Adv-rHSG-GFP. (A) Measuring the expression of rHSG protein by immunocytochemistry; (B) measuring the expression of rHSG protein by western blot analysis; (C and D) histograms of the protein expression of rHSG. ${ }^{* *} \mathrm{P}<0.01$, compared with the control group. 


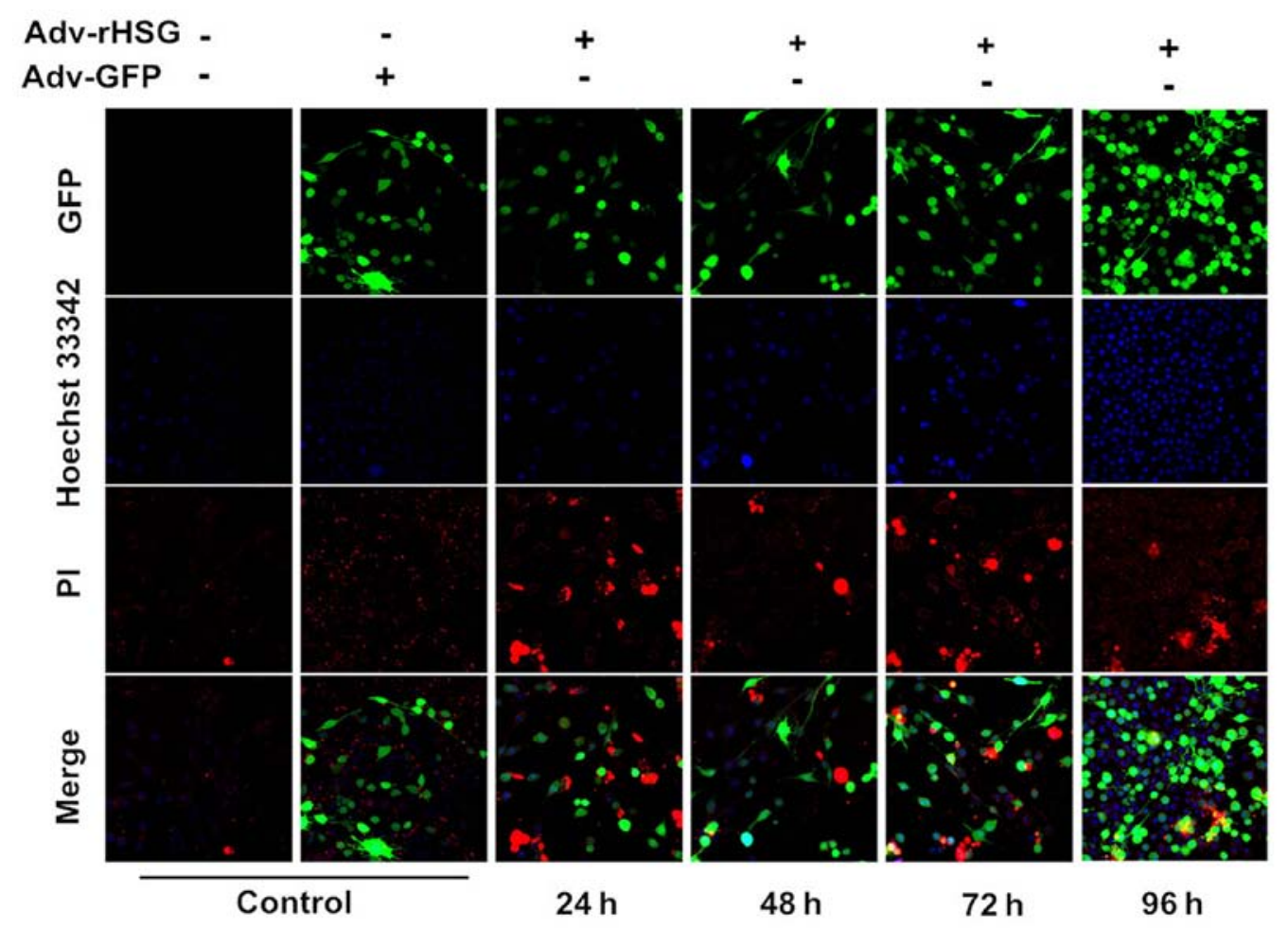

Figure 3. Measuring the effects of rat hyperplasia suppressor gene (rHSG) on the apoptosis of $\mathrm{C} 6$ cells at different time points post-transfection by Hoechst $33342 /$ PI double-staining (x400 magnification).

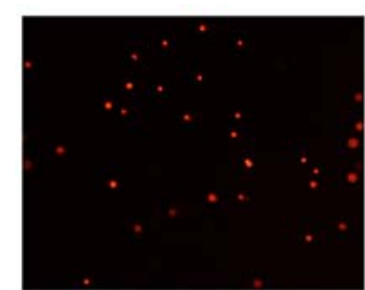

Uninfected

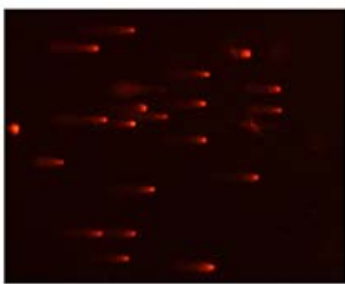

Adv-rHSG-GFP $(48 \mathrm{~h})$

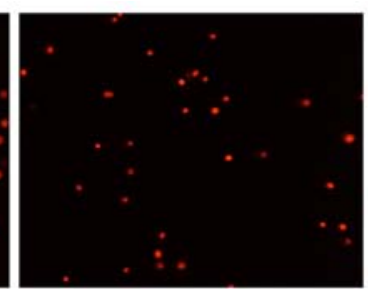

Adv-GFP

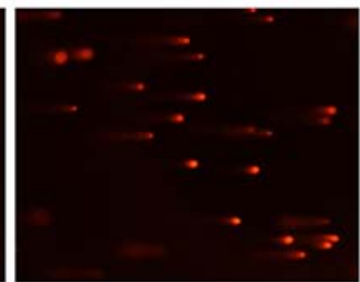

) Adv-rHSG-GFP

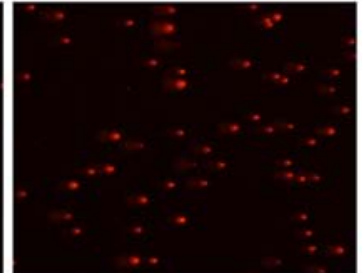

Adv-rHSG-GFP(24h)

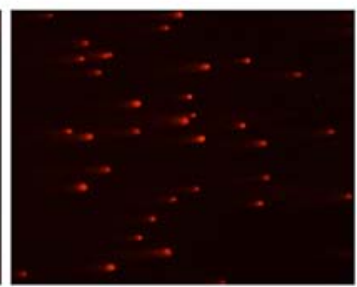

(96h)

Figure 4. Measuring DNA damage in C6 cells induced by rat hyperplasia suppressor gene (rHSG) at different time points post-transfection by comet assay (x400 magnification).

significantly at $48 \mathrm{~h}(\mathrm{P}<0.01, \mathrm{n}=3$; Fig. $5 \mathrm{~A}$ and $\mathrm{B})$. The protein expression of cleaved caspase- 3 in the Adv-rHSG-GFP group was significantly higher than that in the control group or Adv-GFP group $(\mathrm{P}<0.01, \mathrm{n}=3$; Fig. 5C and $\mathrm{D})$; however, no significant differences were observed between the control group and Adv-GFP group ( $\mathrm{P}>0.05)$ (Fig. 5).

rHSG promotes C6 cell apoptosis through the PI3K/Akt and Ras-Raf-MAPK pathways. The results of western blot analysis revealed that the expression of $\mathrm{p}$-Erk1/2 began to decrease at $24 \mathrm{~h}$ post-transfection in the Adv-rHSG-GFP group, although the difference was not statistically significant as compared to the control or Adv-GFP group ( $\mathrm{P}>0.05$; Fig. 6A and B). By contrast, the decrease was statistically significant from the time point of $48 \mathrm{~h}(\mathrm{P}<0.01, \mathrm{n}=3$; Fig. 6A and $\mathrm{B})$. The expression of p-Akt was also evident at $48 \mathrm{~h}$, but not at any of the other time points. In addition, the expression of p-Akt was significantly lower at $48 \mathrm{~h}$ in the Adv-rHSG-GFP group than in the control 


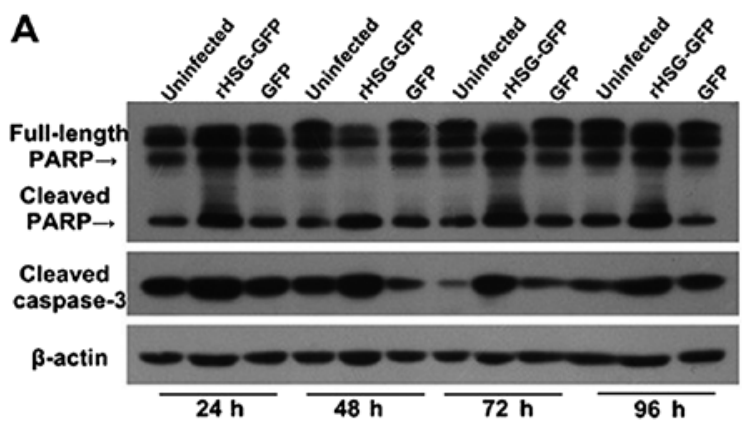

\section{B}

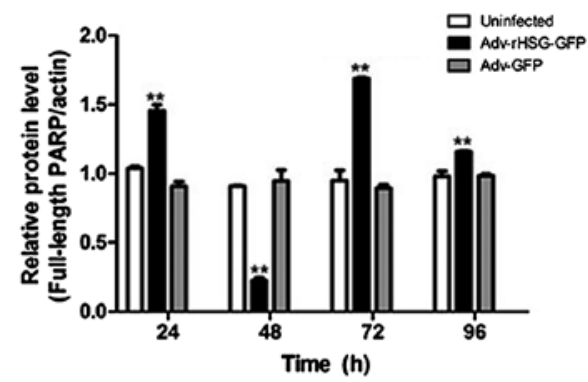

C
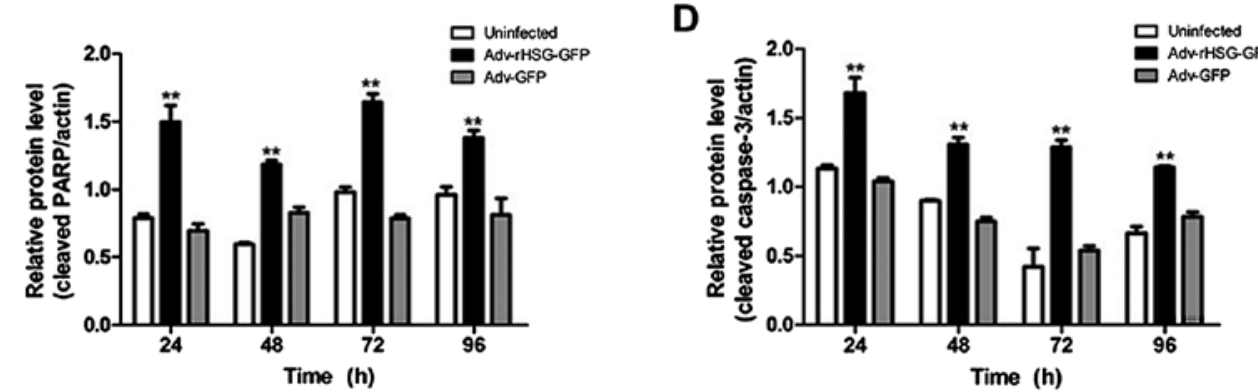

Figure 5. Protein expression of poly(ADP-ribose) polymerase (PARP) and cleaved caspase-3 in the C6 cells transfected with Adv-rat hyperplasia suppressor gene (rHSG)-GFP at different time points. (A) Measuring the expression of PARP and cleaved caspase-3 proteins by western blot analysis; (B-D) histograms showing the protein expression. ${ }^{* *} \mathrm{P}<0.01$, compared with the control group.

A

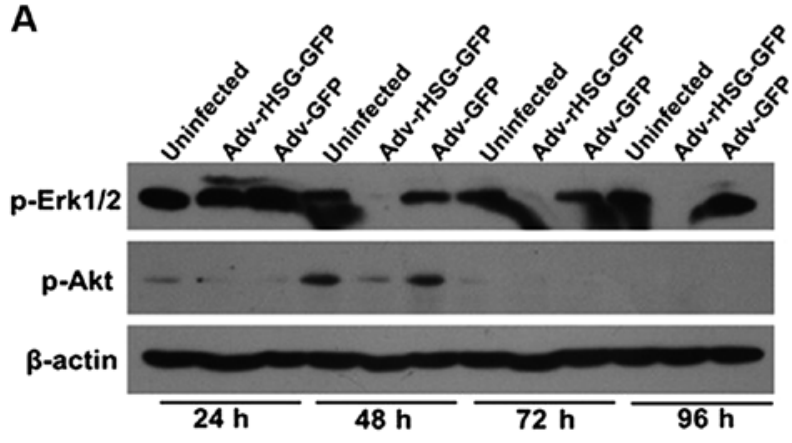

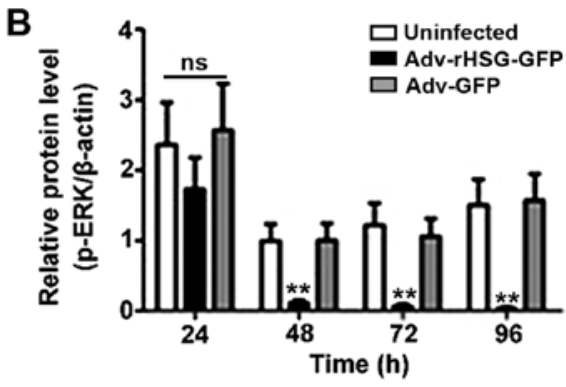

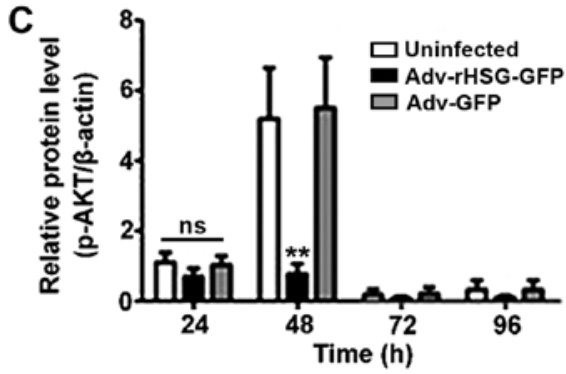

Figure 6. Changes in the levels of p-Erk1/2 and p-Akt in the C6 cells at different time points post-transfection with Adv-rat hyperplasia suppressor gene (rHSG)-GFP. (A) Measuring the expression of p-Erk and p-Akt in each group by western blot analysis; (B and C) histograms showing the protein expression. ${ }^{~} \mathrm{P}<0.01$, compared with the control group; ns, not significant.

or Adv-GFP group $(\mathrm{P}<0.01, \mathrm{n}=3$; Fig. 6A and $\mathrm{C})$; no significant difference was observed between the control and Adv-GFP groups ( $\mathrm{P}>0.05$; Fig. 6 ).

The expression levels of p-Akt and p-Erk1/2 increased after the $\mathrm{C} 6$ cells were stimulated with IGF-1, while the total expression levels of Akt and Erk1/2 were not significantly altered. The expression levels of $\mathrm{p}$-Akt and p-Erk1/2 increased slightly when $1 \mathrm{ng} / \mathrm{ml}$ of IGF-1 was used, and increased significantly when 5 ,
8 and $10 \mathrm{ng} / \mathrm{ml}$ of IGF-1 were used $(\mathrm{P}<0.01, \mathrm{n}=3$; Fig. 7A-D). The expression levels of $\mathrm{p}-\mathrm{Akt}$ and $\mathrm{p}$-Erk $1 / 2$ were the highest when $10 \mathrm{ng} / \mathrm{ml}$ of IGF-1 was used; thus, $10 \mathrm{ng} / \mathrm{ml}$ of IGF-1 was selected as the optimal concentration for the subsequent evaluation of p-Akt and p-Erk1/2 protein expression in the C6 cells. After the $\mathrm{C} 6$ cells were treated with the optimal concentration of IGF-1 for 0, 5, 15, 30 and $60 \mathrm{~min}$, we found that the expression levels of p-Akt and p-Erk1/2 were the highest at the time 


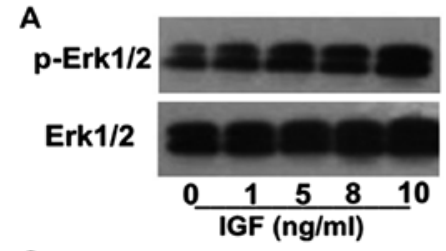

C
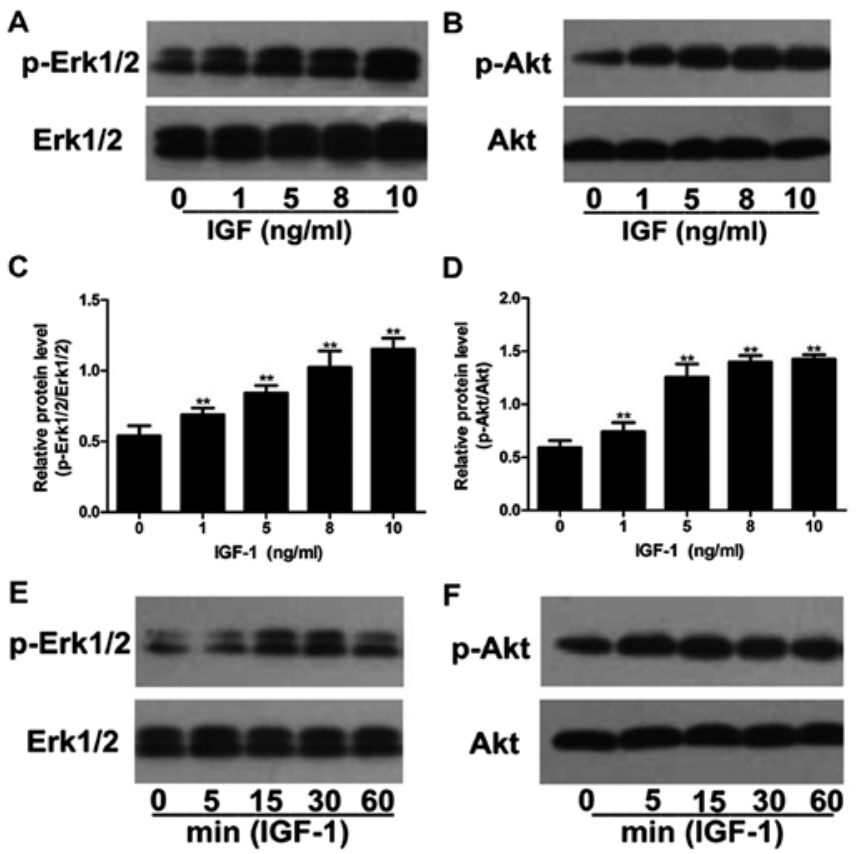

G
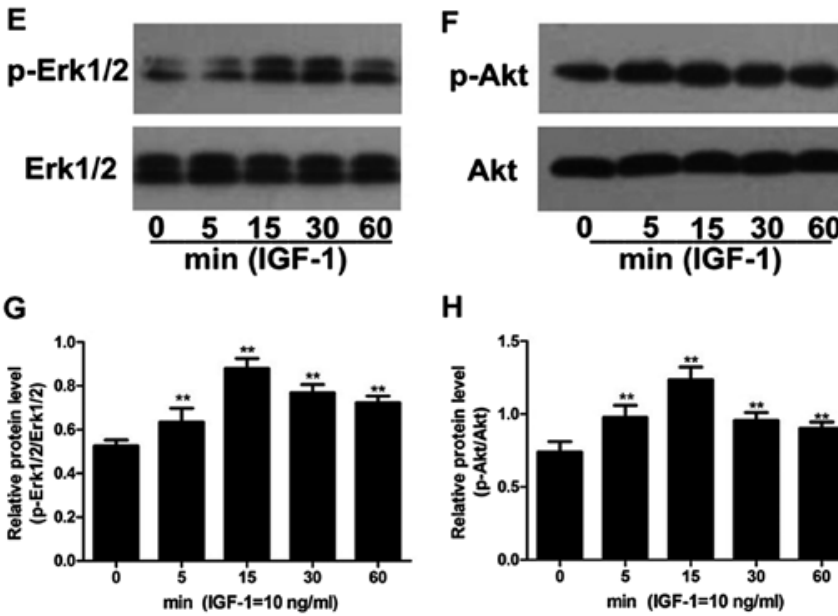

H

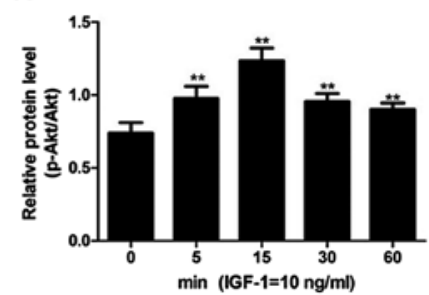

Figure 7. Measuring the expression levels of p-Akt and p-Erk1/2 in the C6 cells induced by insulin-like growth factor (IGF)-1 by western blot analysis (A) p-Erk1/2 protein expression in the C6 cells induced by various concentrations of IGF-1; (B) p-Akt protein expression in the C6 cells induced by various concentrations of IGF-1; (E) p-Erk1/2 protein expression in the C6 cells at different time points post-stimulation with IGF-1; (F) p-Akt protein expression in the $\mathrm{C} 6$ cells at different time points post-stimulation with IGF-1; $(\mathrm{C}, \mathrm{D}, \mathrm{G}$, and $\mathrm{H}$ ) histograms showing the expression of $\mathrm{p}-\mathrm{Akt}$ and $\mathrm{p}$-Erk1/2. ${ }^{* *} \mathrm{P}<0.01$, compared with IGF-1 $=0 \mathrm{ng} / \mathrm{ml}$ group in $(\mathrm{C}$ and $\mathrm{D})$, and with the time point of 0 min in $(\mathrm{G}$ and $\mathrm{H})$.

point of $15 \mathrm{~min}$ (Fig. 7E-H). Therefore, $15 \mathrm{~min}$ was selected as the optimal time point for the following experiments to measure the expression levels of p-Akt and p-Erk1/2 in the C6 cells stimulated with IGF-1.

The expression levels of p-Akt and p-Erk1/2 in the C6 cells stimulated with IGF-1 were significantly higher than the non-stimulated C6 cells $(\mathrm{P}<0.01, \mathrm{n}=3)$. By contrast, the expression levels of $\mathrm{p}$-Akt and p-Erk1/2 did not differ significantly between the control and Adv-GFP groups, but decreased significantly with time in the Adv-rHSG-GFP group $(\mathrm{P}<0.01, \mathrm{n}=3)$. In addition, the expression levels of these two proteins were also significantly lower at each time point post-tranfsection compared with the control group $(\mathrm{P}<0.01, \mathrm{n}=3$; Fig. 8).

\section{Discussion}

rHSG has now been recognized as a hyperplasia suppressor gene. It has been demonstrated that transfection with rHSG promotes the apoptosis of some malignant cell lines, including hepatocellular carcinoma (HCC) cells and the bladder cancer cell lines, T24 and 5637, suggesting that rHSG has substantial pro-apoptotic functions $(22,23)$. However, the effects of rHSG on glioma cell apoptosis have not been reported to date, at least to the best of our knowledge. The most important findings of the present study are that the overexpression of rHSG promoted the apoptosis of C6 cells, which were confirmed by a series of experimental findings. Firstly, a recombined adenovirus system was used to induce the highly efficient expression of rHSG in the C6 cells. Subsequently, several experiments including the observation of the cell nuclei morphological cahnges, DNA damage, and the measurement of the expression of apoptosisrelated factors demonstrated that the rHSG gene significantly promoted the apoptosis of C6 cells. Secondly, the measurement of the expression of p-Akt and p-Erk1/2, two important factors in the Ras-Raf-MAPK and PI3K/Akt pathways, revealed that rHSG significantly inhibited the expression of these two factors. Finally and most importantly, stimulating the cells with IGF-1, a factor that stimulates the Ras-Raf-MAPK and PI3K/Akt pathways, confirmed the association between rHSG and these two pathways, and further provided evidence of the role of the Ras-Raf-MAPK and PI3K/Akt pathways in the apoptosis of C6 cells. These findings suggest that rHSG is of great importance in identifying and treating many malignant cells, including glioma cells, as well as in apoptosis in cell proliferative disorders.

Previous studies have demonstrated that PARP is a substrate of caspase-3; therefore the activation of caspase-3 induces cell apoptosis, and thus PARP is also known as 'death substrate' (24-26). PARP is associated with the repair of DNA and the integrity of genes. Caspase-3 recognizes the DVCD sequences in PARP following activation and thus cleaves PARP, which disrupts the normal functions of PARP. Altered PARP induces the activity of endonuclease that is negatively regulated by PARP, and thus finally degrades the DNA between nucleosomes and consequently induces cell apoptosis (27-32). The findings of the present study demonstrating that the transfection of C6 cells with rHSG induced cell apoptosis also confirmed these theories. However, we also observed that the expression of cleaved PARP increased during this process. The expression of full-length PARP was also increased at 24, 72 and $96 \mathrm{~h}$ post-transfection, suggesting that rHSG induces the activation of two pathways with opposite effects, namely one pro-apoptotic pathway and one anti-apoptotic pathway. We hypothesized that when rHSG induced DNA damage, in order to survive, the cells produced PARP for repairing the DNA. rHSG also activated the pro-apoptotic pathway, which activated the critical downstream factor, caspase-3, to cleave corresponding PARP, and thus the expression of cleaved PARP increased significantly. In the present study, we found that the expression of cleaved PARP increased considerably, while the expression of full-length PARP decreased significantly at $48 \mathrm{~h}$ post-transfection compared to the control and Adv-GFP groups, and this is the time point at which the rHSG protein level was the highest. These findings suggested that the DNA repairing effects of PARP at this time point were effectively inhibited, and the C6 cells were mainly in the state of apoptosis induced by rHSG.

In order to investigate further the effects of rHSG on the pathways of anti-/pro-apoptosis in the cells, we measured the levels of p-Akt and p-Erk1/2, which are the two critical factors 

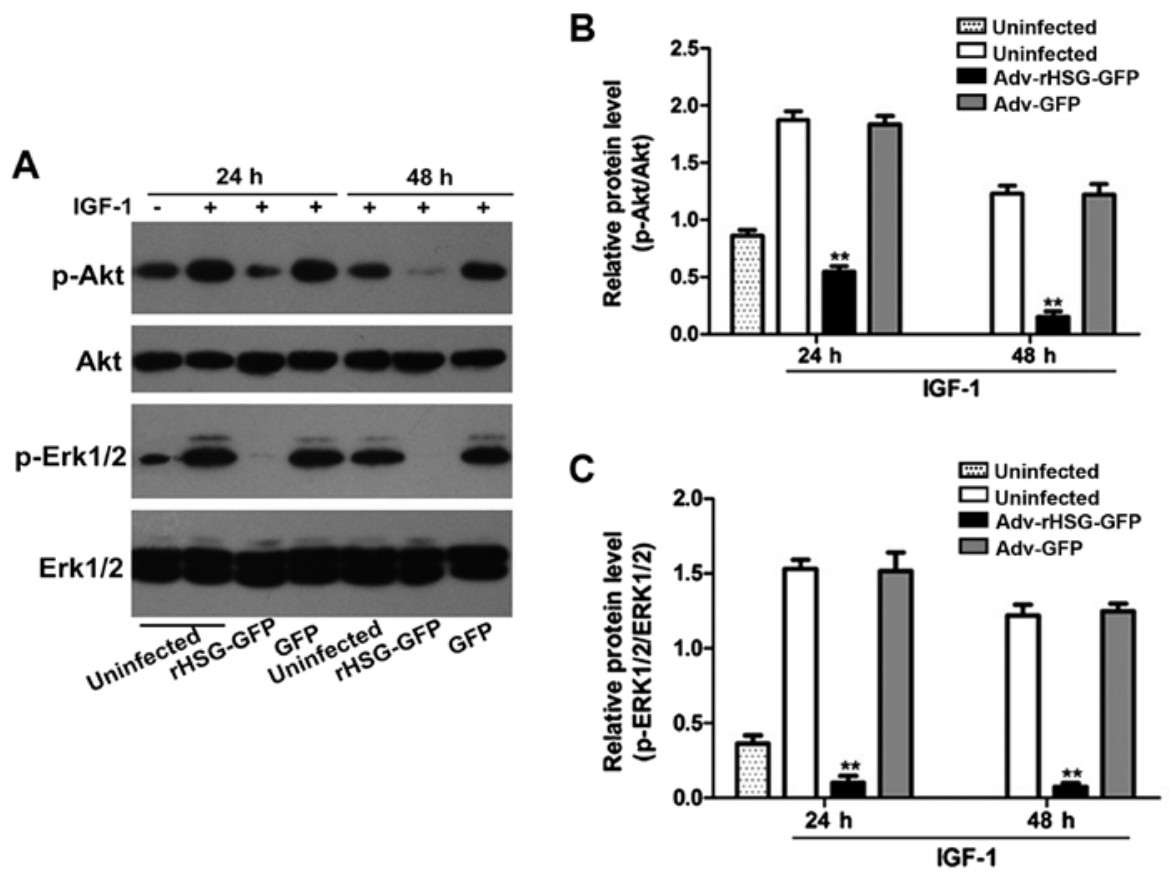

Figure 8. Measuring the effects of rat hyperplasia suppressor gene (rHSG) overexpression on the phosphorylation of Akt and Erk1/2 induced by insulin-like growth factor (IGF)-1 by western blot analysis. (A) Effects of rHSG overexpression on p-Akt and p-Erk1/2 expression induced by IGF-1; (B and C) histograms showing the expression of $\mathrm{p}$-Akt and $\mathrm{p}$-Erk $1 / 2 .{ }^{* *} \mathrm{P}<0.01$, compared with the uninfected group and Adv-GFP group.

in the Ras-Raf-MAPK and PI3K/Akt pathways. It has been demonstrated that inhibition of the MAPK pathway is also a factor that causes cell apoptosis; it has been shown that in pancreatic cancer cells and epithelial cells, the increased expression of $\mathrm{p}$-Erk1/2 induced the phosphorylation of BAD protein, and thus inhibited cell apoptosis $(33,34)$. The PI3K/Akt pathway is a classic pathway that inhibits cell apoptosis and maintains cell survival. PI3K interacts with phosphoinositide-dependent protein kinase (PDK) following activation, which activates Akt and thus increases the transcription of the downstream survival-related genes to maintain cell survival, or induce the phosphorylation of BAD protein to inhibit cell apoptosis (35). The findings of the present study demonstrated that the protein expression levels of Erk1/2 and Akt decreased significantly at $48 \mathrm{~h}$ post-rHSG transfection, at which time point the rHSG level was the highest, but no significant decrease was observed at $24 \mathrm{~h}$ post-transfection. Therefore, we introduced IGF-1, a factor that stimulates the PI3K/Akt and MAPK pathways, to stimulate the cells. The stimulating effects of IGF-1 were firstly identified on the PI3K/Akt pathway $(36,37)$, and further studies also found that IGF-1 activates the MAPK pathway as well $(38,39)$. During the activation of these pathways, IGF plays an important role in anti-apoptosis $(40,41)$. In the present study, Adv-rHSG-GFP was used to transfect the C6 cells, after which IGF-1 was used to activate the Erk1/2 and PI3K/Akt pathways in these cells. The expression levels of $\mathrm{p}$-Akt and $\mathrm{p}$-Erk1/2 increased significantly to protect the cells from apoptosis. However, the expression levels of p-Akt and p-Erk1/2 were significantly lower than the level in the control group at the corresponding time point of 24 and $48 \mathrm{~h}$ post-transfection. These expression levels decreased further with time. These findings indicated that the combined use of rHSG and IGF-1 significantly promoted the pro-apoptotic effects of rHSG on
C6 cells. We hypothesized that the exogenous cell protective factor, IGF-1, decreased the repair and protective effects of the cells, and thus enhanced the pro-apoptotic effects of rHSG on the $\mathrm{C} 6$ cells. These findings suggested that the activation of the Erk1/2 and Akt pathways also protected the cells and inhibied the apoptosis induced by rHSG.

In conclusion, these findings suggest that the overexpression of rHSG promotes the apoptosis of rat glioma cells, and the molecular mechanisms involve the PI3K/Akt and Ras-RafMAPK pathways. The Ras-Raf-MAPK pathway may play a dominant role in the effects, suggesting that rHSG may be a potential target for the treatment of gliomas.

The present study provided additional evidence on the effects of rHSG on rat glioma (42), which is also important for further studies on glioma. Changes in caspase-3 expression were also observed in the present study. However, further studies are required to investigate further the molecular mechanisms involved in the pro-apoptotic effects of rHSG on glioma cells, particularly the other two pathways with caspase-3 involvement.

\section{Acknowledgements}

This study was supported by the the Ningxia Hui Autonomous Region Education Department Foundation (no. NGY2011054), and the Natural Science Foundation Key Project of the Ningxia Hui Autonomous Region (no. NZ13129).

\section{References}

1. Fuller GN and Scheithauer BW: The 2007 Revised World Health Organization (WHO) classification of tumours of the central nervous system: newly codified entities. Brain Pathol 17: 304-307, 2007. 
2. Reifenberger G, Hentschel B, Felsberg J, Schackert G, Simon M, Schnell O, Westphal M, Wick W, Pietsch T, Loeffler M, et al; German Glioma Network: Predictive impact of MGMT promoter methylation in glioblastoma of the elderly. Int J Cancer 131: 1342-1350, 2012.

3. Grossman SA, Ye X, Piantadosi S, Desideri S, Nabors LB, Rosenfeld M and Fisher J; NABTT CNS Consortium: Survival of patients with newly diagnosed glioblastoma treated with radiation and temozolomide in research studies in the United States. Clin Cancer Res 16: 2443-2449, 2010.

4. Jemal A, Bray F, Center MM, Ferlay J, Ward E and Forman D: Global cancer statistics. CA Cancer J Clin 61: 69-90, 2011.

5. Chinese Glioma Cooperative Group (CGCG), Chinese Glioma Atlas (CGGA): Chinese Glioma Molecular Guidelines. Chin J Neurosurg 30: 435-444, 2014

6. Candolfi M, Kroeger KM, Muhammad AK, Yagiz K, Farrokhi C, Pechnick RN, Lowenstein PR and Castro MG: Gene therapy for brain cancer: combination therapies provide enhanced efficacy and safety. Curr Gene Ther 9: 409-421, 2009.

7. Nakada M, Niska JA, Tran NL, McDonough WS and Berens ME: EphB2/R-Ras signaling regulates glioma cell adhesion, growth, and invasion. Am J Pathol 167: 565-576, 2005.

8. Jeon BN, Yoo JY, Choi WI, Lee CE, Yoon HG and Hur MW: Proto-oncogene FBI-1 (Pokemon/ZBTB7A) represses transcription of the tumor suppressor $R b$ gene via binding competition with Sp1 and recruitment of co-repressors. J Biol Chem 283: 33199-33210, 2008.

9. Westphal M and Lamszus K: The neurobiology of gliomas: from cell biology to the development of therapeutic approaches. Nat Rev Neurosci 12: 495-508, 2011

10. Chen KH, Guo X, Ma D, Guo Y, Li Q, Yang D, Li P, Qiu X, Wen S, Xiao RP and Tang J: Dysregulation of HSG triggers vascular proliferative disorders. Nat Cell Biol 6: 872-883, 2004.

11. Guo X, Chen KH, Guo Y, Liao H, Tang J and Xiao RP: Mitofusin 2 triggers vascular smooth muscle cell apoptosis via mitochondrial death pathway. Circ Res 101: 1113-1122, 2007.

12. Wu M, Chen Q, Li D, Li X, Li X, Huang C, Tang Y, Zhou Y, Wang D, Tang K, et al: LRRC4 inhibits human glioblastoma cells proliferation, invasion, and proMMP-2 activation by reducing SDF-1 alpha/CXCR4-mediated ERK1/2 and Akt signaling pathways. J Cell Biochem 103: 245-255, 2008.

13. Wu M, Huang C, Li X, Li X, Gan K, Chen Q, Tang Y, Tang K, Shen S and Li G: LRRC4 inhibits glioblastoma cell proliferation, migration, and angiogenesis by downregulating pleiotropic cytokine expression and responses. J Cell Physiol 214: 65-74, 2008.

14. Kunapuli P, Kasyapa CS, Hawthorn L and Cowell JK: LGII, a putative tumor metastasis suppressor gene, controls in vitro invasiveness and expression of matrix metalloproteinases in glioma cells through the ERK1/2 pathway. J Biol Chem 279: 23151-23157, 2004.

15. Bhaskara VK, Sundaram C and Babu PP: pERK, pAkt and pBad: a possible role in cell proliferation and sustained cellular survival during tumorigenesis and tumor progression in ENU induced transplacental glioma rat model. Neurochem Res 31: 1163-1170, 2006.

16. Cuevas P, Diaz-González D, Carceller F and Dujovny M: Dual blockade of mitogen-activated protein kinases ERK-1 (p42) and ERK-2 (p44) and cyclic AMP response element binding protein (CREB) by neomycin inhibits glioma cell proliferation. Neurol Res 25: 13-16, 2003

17. Belsey MJ, Davies AR, Witchel HJ and Kozlowski RZ: Inhibition of ERK and JNK decreases both osmosensitive taurine release and cell proliferation in glioma cells. Neurochem Res 32: 1940-1949, 2007

18. Betti M, Minelli A, Canonico B, Castaldo P, Magi S, Aisa MC, Piroddi M, Di Tomaso V and Galli F: Antiproliferative effects of tocopherols (vitamin E) on murine glioma C6 cells: homologue-specific control of PKC/ERK and cyclin signaling. Free Radic Biol Med 41: 464-472, 2006.

19. McDaid HM, Lopez-Barcons L, Grossman A, Lia M, Keller S, Pérez-Soler R and Horwitz SB: Enhancement of the therapeutic efficacy of taxol by the mitogen-activated protein kinase kinase inhibitor CI-1040 in nude mice bearing human heterotransplants. Cancer Res 65: 2854-2860, 2005.

20. Knobbe CB, Trampe-Kieslich A and Reifenberger G: Genetic alteration and expression of the phosphoinositol-3-kinase/Akt pathway genes PIK3CA and PIKE in human glioblastomas. Neuropathol Appl Neurobiol 31: 486-490, 2005

21. Gao P, Jiang S, Guo H, Yang Q, Fang Z, Zhao W and Shen B: Expression and mechanism of rat hyperplasia suppressor gene suppressing growth of rat C6 glioma cells. J Third Mil Med Univ 36: 381-385, 2014
22. Jin B, Fu G, Pan H, Cheng X, Zhou L, Lv J, Chen G and Zheng S: Anti-tumour efficacy of mitofusin-2 in urinary bladder carcinoma. Med Oncol 28 (Suppl 1): S373-S380, 2011

23. Wang W, Lu J, Zhu F, Wei J, Jia C, Zhang Y, Zhou L, Xie H and Zheng S: Pro-apoptotic and anti-proliferative effects of mitofusin-2 via Bax signaling in hepatocellular carcinoma cells. Med Oncol 29: 70-76, 2012

24. GagnéJP,Moreel X,GagnéP,Labelle Y,Droit A,Chevalier-ParéM, Bourassa S, McDonald D, Hendzel MJ, Prigent C and Poirier GG: Proteomic investigation of phosphorylation sites in poly(ADPribose) polymerase-1 and poly(ADP-ribose) glycohydrolase. J Proteome Res 8: 1014-1029, 2009.

25. Reed AM, Fishel ML and Kelley MR: Small-molecule inhibitors of proteins involved in base excision repair potentiate the anti-tumorigenic effect of existing chemotherapeutics and irradiation. Future Oncol 5: 713-726, 2009.

26. Atorino L, Di Meglio S, Farina B, Jones R and Quesada P: Rat germinal cells require PARP for repair of DNA damage induced by gamma-irradiation and $\mathrm{H}_{2} \mathrm{O}_{2}$ treatment. Eur J Cell Biol 80: 222-229, 2001

27. Thornberry NA and Lazebnik Y: Caspases: enemies within. Science 281: 1312-1316, 1998.

28. Kato J, Kuwabara Y, Mitani M, Shinoda N, Sato A, Toyama T, Mitsui A, Nishiwaki T, Moriyama S, Kudo J and Fujii Y: Expression of survivin in esophageal cancer: correlation with the prognosis and response to chemotherapy. Int J Cancer 95: 92-95, 2001.

29. Altieri DC: Survivin, versatile modulation of cell division and apoptosis in cancer. Oncogene 22: 8581-8589, 2003.

30. Beardsmore DM, Verbeke CS, Davies CL, Guillou PJ and Clark GW: Apoptotic and proliferative indexes in esophageal cancer: predictors of response to neoadjuvant therapy [corrected]. J Gastrointest Surg 7: 77-86, 2003.

31. Kelly RJ, Lopez-Chavez A, Citrin D, Janik JE and Morris JC: Impacting tumor cell-fate by targeting the inhibitor of apoptosis protein survivin. Mol Cancer 10: 35, 2011

32. Tamm I, Wang Y, Sausville E, Scudiero DA, Vigna N, Oltersdorf T and Reed JC: IAP-family protein survivin inhibits caspase activity and apoptosis induced by Fas (CD95), Bax, caspases, and anticancer drugs. Cancer Res 58: 5315-5320, 1998

33. Boucher MJ, Morisset J, Vachon PH, Reed JC, Lainé J and Rivard N: MEK/ERK signaling pathway regulates the expression of $\mathrm{Bcl}-2, \mathrm{Bcl}-\mathrm{X}(\mathrm{L})$, and $\mathrm{Mcl}-1$ and promotes survival of human pancreatic cancer cells. J Cell Biochem 79: 355-369, 2000.

34. Buder-Hoffmann S, Palmer C, Vacek P, Taatjes D and Mossman B: Different accumulation of activated extracellular signal-regulated kinases (ERK 1/2) and role in cell-cycle alterations by epidermal growth factor, hydrogen peroxide, or asbestos in pulmonary epithelial cells. Am J Respir Cell Mol Biol 24: 405-413, 2001.

35. Neri LM, Borgatti P, Capitani S and Martelli AM: The nuclear phosphoinositide 3-kinase/AKT pathway: a new second messenger system. Biochim Biophys Acta 1584: 73-80, 2002.

36. Ueki K, Fruman DA, Brachmann SM, Tseng YH, Cantley LC and Kahn CR: Molecular balance between the regulatory and catalytic subunits of phosphoinositide 3-kinase regulates cell signaling and survival. Mol Cell Biol 22: 965-977, 2002.

37. Wymann MP and Pirola L: Structure and function of phosphoinositide 3-kinases. Biochim Biophys Acta 1436: 127-150,1998.

38. Khokhlatchev AV, Canagarajah B, Wilsbacher J, Robinson M, Atkinson M, Goldsmith E and Cobb MH: Phosphorylation of the MAP kinase ERK2 promotes its homodimerization and nuclear translocation. Cell 93: 605-615, 1998.

39. Párrizas M, Saltiel AR and LeRoith D: Insulin-like growth factor 1 inhibits apoptosis using the phosphatidylinositol 3'-kinase and mitogen-activated protein kinase pathways. J Biol Chem 272: 154-161, 1997.

40. Kenchappa P, Yadav A, Singh G, Nandana S and Banerjee K: Rescue of TNFalpha-inhibited neuronal cells by IGF-1 involves Akt and c-Jun N-terminal kinases. J Neurosci Res 76: 466-474, 2004

41. Bencomo E, Pérez R, Arteaga MF, Acosta E, Peña O, Lopez L, Avila J and Palumbo A: Apoptosis of cultured granulosa-lutein cells is reduced by insulin-like growth factor I and may correlate with embryo fragmentation and pregnancy rate. Fertil Steril 85 : 474-480, 2006

42. Gao P, Wang Z, Zhang B, Zou Y, Guo H, Liu H, Yang Q, Fang Z, Jiang S, Shen B, et al: Suppression of C6 gliomas via application of rat hyperplasia gene. Int J Biol Markers 29: e411-e422, 2014. 